\title{
Family Aware TV Program and Settings Recommender
}

\author{
Thyagaraju GS \\ Research Scholar (VTU), \\ Dept Of CSE, SDMCET, Dharwad -580 002, \\ Karnataka ,India.
}

\author{
Umakant P Kulkarni \\ Professor \\ Dept of CSE, SDMCET, Dharwad -580 002, \\ Karnataka, India.
}

\begin{abstract}
In this paper we are proposing a design of TV program and settings recommendation engine utilizing contextual parameters like personal, social, temporal, mood and activity. In addition to the contextual parameters the system utilize the explicit or implicit user ratings and watching history to resolve the conflict if any while recommending the services .The System is implemented exploiting AI techniques ( like ontology, fuzzy logic ,Bayesian classifier and Rule Base) , RDBMS and SQL Query Processing . The motivation behind the proposed work is i) to improve the user's satisfaction level and ii) to improve the social relationship between user and TV. The context aware recommender utilizes social context data as an additional input to the recommendation task alongside information of users and tv programs. We have analyzed the recommendation process and performed a subjective test to show the usefulness of the proposed system for small families.
\end{abstract}

\section{General Terms}

Artificial Intelligence, Context Aware Computing, Ubiquitous Systems.

\section{Keywords}

Ubiquitous, context, recommendation engine, conflict, context aware tv, family preference, role, age, social status, favorite program, automatic, fuzzy logic, mood, activity

\section{INTRODUCTION}

The advancement of pervasive computing, sensors technology and the wide deployment of wireless communication (RFID, BlueTooth, WiFi, etc.,) and the availability of artificial intelligence techniques like RuleBase,Bayesian Network ,Fuzzy logic and Neural Networks is motivating many researchers across the world to improve the intellectual and social relationship between man and machine. This trend has resulted in an explosive growth in computing systems and applications that impact all aspects of our life.

Digital TVs are one of the most popular consumer products in every home around the world, and have evolved such that they can now provide personalized and context aware services to users. Due to their popularity, various technologies have been developed that contribute to making the system even more Intelligent. For example, electronic program guides (EPGs) provide users with program schedules and detailed information that enables them to search for desired programs from among a number of available programs, and the TV Anytime Forum specifies the metadata of digital TV. Consequently, additional technologies and applications have become available for consumers, and systems have become more intelligent by exploiting user information such as profiles, history, feedback, preferred program schedule, and the real-time program information offered by EPG and TV Anytime specifications. However, although these technologies have improved how well digital TVs respond to users, they must also take into account group of users that may want simultaneous access to the device, in addition to the above technological achievements. $[1,2,3,4,5]$

The goal of our research is to develop a recommendation engine for TV which facilitates the system to act autonomously in the way the family members desires in single and multiuser environment. We are the first to use the hierarchical structure of family effectively in recommendation engine. We formulate the service adaptation process by using artificial intelligence techniques fuzzy logic, Bayesian classifier and the utility theory in order to recommend appropriate service with respect to the context. The recommendation engine has been designed focusing on user's social context like personalization, role, age, interest, time, location, mood and activity. The prototype is tested for live cable TV in a living room and the data obtained by surveying different families through interviews and observations. The motivation or goal is captured in the following main research question: "How can one develop a family friend intelligent TV system?". Fig1 illustrates a scenario where in the TV is thinking how to satisfy the family.

The remainder of this paper is structured as follows. Section 2 gives a different research efforts carried out over the years in context aware recommendation systems with emphasis on user context. Section 3 discusses the Modeling and design of the proposed work. Section 4 gives a detail description of proposed algorithms. Section 5 gives the implementation details .Section 6 gives the Experimental Data and Result analysis. Section 7 concludes with future work.

\section{RELATED WORKS}

Socialization and Personalization of consumer devices is an active research topic .A general definition of socialization is to make someone behave in a way that is acceptable to society. Personalization is "understanding the needs of each individual and helping satisfy a goal that efficiently and knowledgeably addresses each individual's need in a given context ". Personalization and Socialization has realy gained importance with always connected services in the context aware applications. Context aware applications and services use context information to provide relevant services to the user and task at hand $[6,7,8,9,10,11,12]$. 


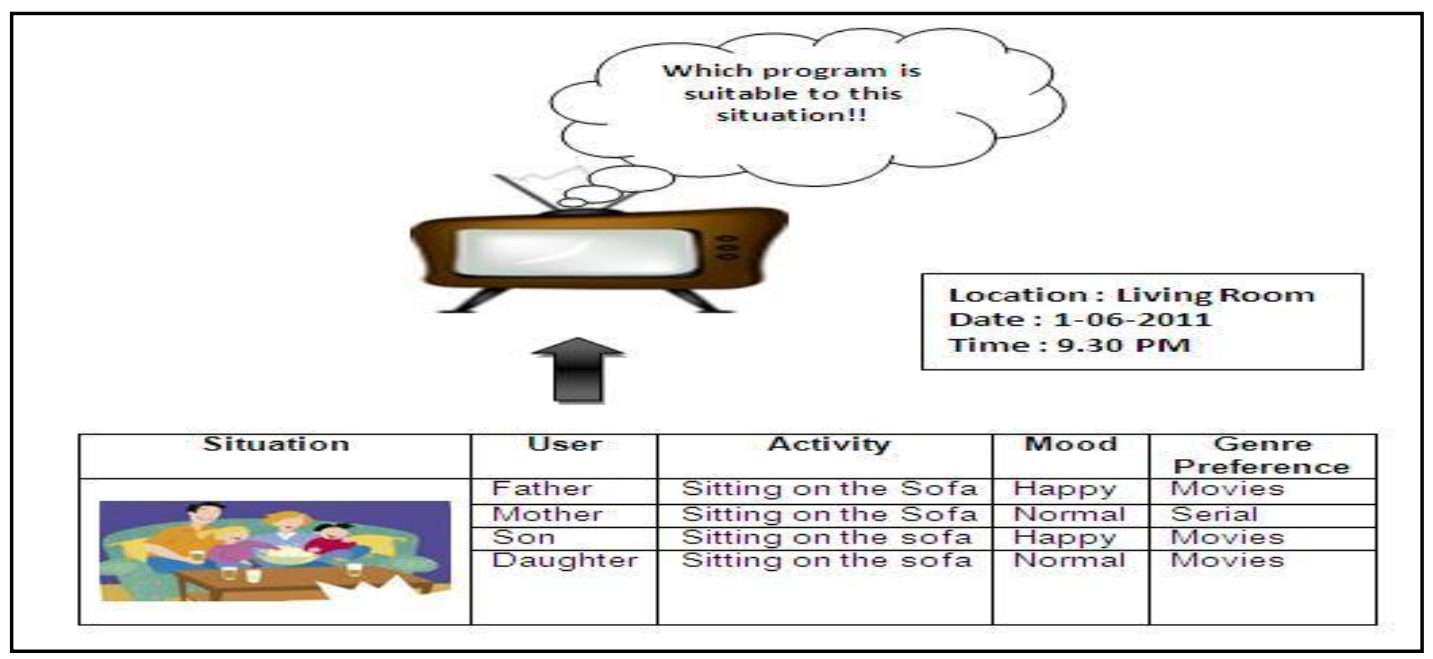

Fig1: Family watching television

Recommender systems are intimately related to personalized services. In theory recommender systems provide the underlying implementation of personalization in practice, recommendation and personalization often combine to one .Recommendation is usually cast as a prediction problem: given a limited set of ratings of items by users ,predict the values of missing ratings .In that scenario a recommender system first collects user rating data, computes unknown rating predictions and finally recommends the highest rated items from the predicted set .Naturally the more rating data is accumulated for a user or an item, the better the recommendations become for them. Recommenders are commonly implemented using collaborative filtering methods .In pure collaborative filtering only the ratings of items by users are used in providing recommendations. Other recommender systems analyze the content to provide recommendations. The context aware recommender utilizes context data as an additional input to the recommendation task, alongside information of users and items $[13,14,15,16,17,18,19,20,21,22,23,24]$.

Over the last decade, most research, aimed on, have been done on consumer product like smart TV, smart car, smart homes, intelligent office, interactive tourist guide, intelligent environment, ambient intelligence etc. Artur Lugmayr in his research articles stresses the need of integration of Natural interaction, personalization, smart metadata, wireless technology, ubiquitous systems, pervasive computation, and embedded systems. To satisfy the entertainment-hungry consumer, more and more advanced home entertainment (HE) systems and facilities are required to provide interactive and smart leisure content. [25, 26, 27, 28].

Personalized TV services are a prime application of recommender systems as rating data is often readily available. Ray van Brandenburg in his master thesis focuses on the paradox between personalization and the concurrent use of television by multiple users.

MARILYN ("Multimodal Avatar Responsive Live News Caster") is a prototype for business television (BTV). Use of virtual human avatars for intelligent interaction with digital TV.
MARILYN motivates an analysis of the requirement engineering for IITV and suggests a framework deploying natural language understanding, speech recognition, and an experience-based approach to decision support for establishing an IITV platform[5].

iDTV("Intelligent digital TV") is a future digital TV with intelligence which can automatically provide user personalized services for each audience. For the user personalized services, the iDTV should recognize audiences in real-time. In this paper, we define a novel structure of the iDTV and propose a real-time person identification system in the iDTV that analyzes captured images and recognizes audiences [1].

Unlike the selections for a single user, selections associated with a group of users are more complex, thus making it far more difficult to deliver the best solution in terms of diverse user preferences and levels of satisfaction. Only limited research, aimed at realizing a future Interactive Context Aware TV, has contributed to resolving these limitations. $\mathrm{Yu}$ and Zhou proposed a group recommendation algorithm that merges individual user profiles into a group profile based on the distance minimization algorithm. However, this merging only included individual preferences as group characteristics were omitted in the final recommendations. In other studies, Mast hoff discussed various selection strategies based on social theory and found that users were not dedicated to a specific selection method, but may use different strategies in selecting programs. The universal controller, proposed by Yoon and Woo, allowed users to intuitively control a smart TV as a group, but it required users to be actively involved in the control [16, 20, 23, 30, 46, 47]. Choonsung Shin and Woontack Woo proposed a series of socially aware program recommender for multiple viewers of digital TV using different concepts like linear selection method to Bayesian. SongJie Gong proposed a collaborative filtering recommendation algorithm based on user clustering and item clustering .Manos Papagelis, Dimitris Plexousakis have done qualitative analysis of user based and item based prediction algorithms for recommendation agents.[6,24,31,32,33,34].As such, even though various strategies have been developed in attempts to assist the selection of TV programs for a group of users, individual preferences and at most user priority are mainly 
used in generating lists of preferred programs and in final program selection. In addition to the personalization our proposed system uses societal structure of family to make TV more social and user friendly.

In comparison with the previous works the major contribution of this paper can be summarized as follows:

1. Design of Recommendation engine utilizing the contextual parameters like Personal(Age ,Name ) ,Temporal (time ,date, season), social (role ,dominant factor ,age factor ) activity (reading , talking , meeting) , Mood (Normal ,Excited,Happy,Relax,Sad,Disturbed,Dippressed) and Schedule Agendas in addition to the user ratings for the services.

2. Quantifying Social Roles of individual Family members.

3. Exploitation of hybrid ontology, Fuzzy system, Bayesian Networks and the utility theory (watching history and context history) for modeling and implementation.

4. Context storage and retrieval using relational database and SQL query processing.

5. Executing Actions using simple if then else rules base.

\section{DESIGN AND MODELING OF THE PROPOSED SYSTEM}

Our main objective is to embed the user's personal feeling and social (family) awareness in the recommendation system. Here the purpose of TV service refers to the subjective reasons why certain programs are viewed in certain social situations by certain persons. Our hypothesis is that TV programs has different purpose for different family members in different situations and to truly personalize the TV program offering, the TV service should model the purpose of TV program to its viewer .In real-time situations users preferences are truly influenced by the personal feeling (mood) and social relationships between the members .Therefore we postulate that social and personal context or situations encode additional structure that can be utilized to improve qualitative recommendation performance. In this setting the purpose of tv viewing reflects and represents the commonalities between the tv program, the family members and the situation of viewing.

\section{Modeling three domains :}

The problem is associated with three domains of data: family data, context data, and tv content data.

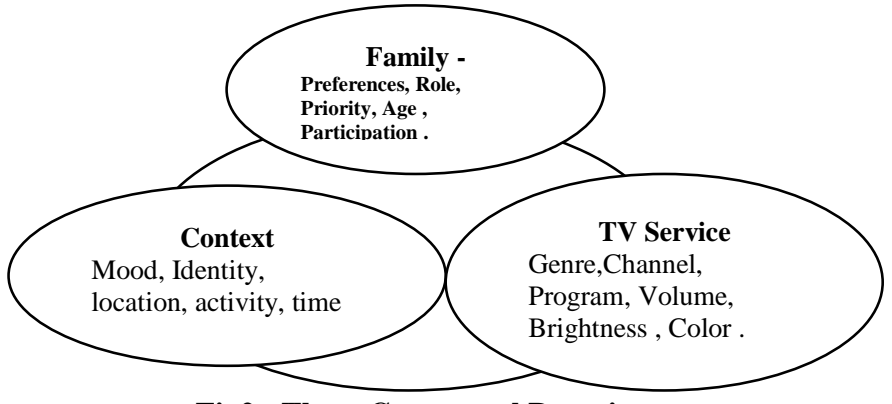

Fig2 : Three Conceptual Domains
The immediate research question arises as to how three domains should be best combined for learning of tv program and settings purpose. Figure 2 illustrates the three different domains of input features for context ware personal tv services:

1. Family domain has information about user profiles, social roles and relationships.

2. Context domain represents the situations that link users with tv; and

3. TV content domain encapsulates tv programs and settings metadata and their descriptors.

The three domains are conceptually orthogonal and as such act as independent sources of data for the tv service recommendation problem. From these three data sources the tv service recommender aims to predict purposeful service selections, given the past behavior of the member in different situations, based on the user and context features. Here we emphasize the fact that the recommendations are not only to be personalized but also to be situationalized according to the learned purposes of tv programs and settings in similar past situations. In practice, to avoid requiring to store and process the full feature set for each prediction the recommenders learn a context aware and personal service purpose model, which is more compact than the full dataset but retains a desired prediction accuracy.

\subsection{Family Modeling:}

Family is a established group of members of mixed attributes like age, sex and role .Families may be small, big or joint families.

A Family is a 5 tuple <Fm, Rel, N, Mt, Reg> --- (1)

Where Fm $=$ Set of Family members,

Rel = Religion /Caste/Community,

$\mathrm{N}=$ Nationality,

$\mathrm{Mt}=$ Mother Tongue and

Reg $=$ Region of residence (State/Province).

Where each Fm is 4 tuple

< Name, Role, Age, Dependency, Preference>--(2)

Even though family is a small sized group but it is a source of multiple conflicts. The number of conflicts is directly proportional to number of social factors like family size , age differences ,role differences ,education, etc., Consider a situation where in Father, Mother, Son and Daughter are watching TV .In such situation since Father is more dominant his preference will have more weightage as compared to the other.(as per the survey on 50 indian nuclear families). In most of the Indian families it is the member of the family with high dominance who resolves the conflict whenever occurs. Fig3 illustrates the member's hierarchy in different small families .While recommending a service to family members when watching TV it is very important to consider the social dominance factor (Sdf) of the member. 


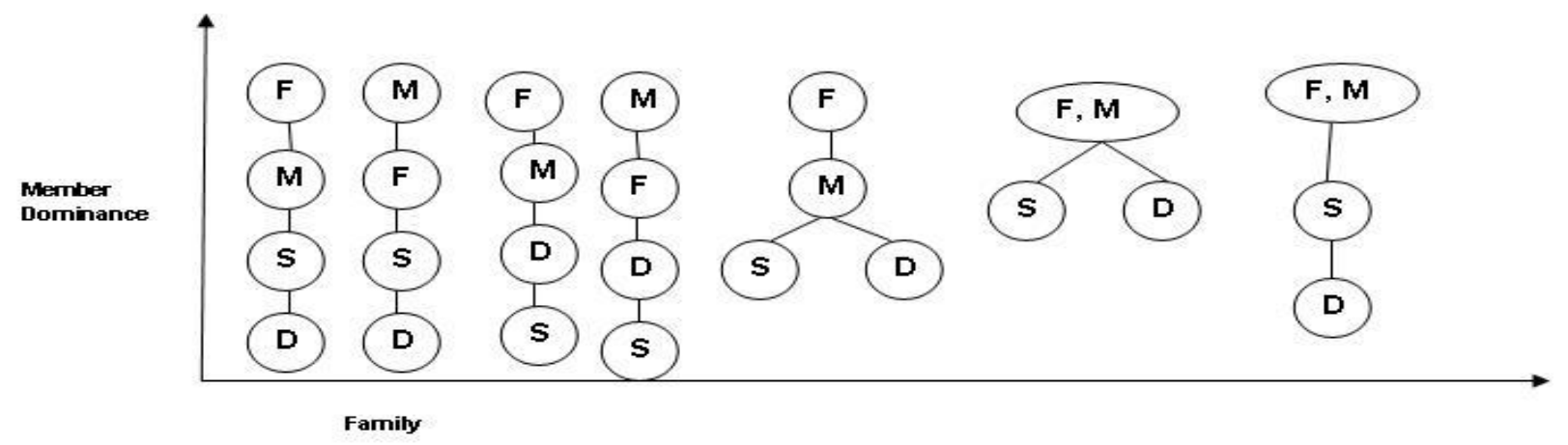

Fig3 : Hierarchy of Family members

Definition: Social dominance factor (SDF) is a quantified value $[0,1]$ representing the user dominance in a given family. The dominance of member depends on

i) the role of the member say Father, Mother ,Son, Daughter ,GrandFather ,GrandMother,etc...

ii) family dependency on the member

iii) age of the person

Each role of the family can be quantified in terms of role factor between $[0,1]$. For example in our work we have considered role factor (weight) value 1 for parents and 0.5 for children based on hierarchy. In most of the families it is the parents (either F, Mother or Both) who run the show. They are the source of love, affection, income and management. Naturally the entire family depends on them .We have quantified the family dependency to 0 or 1 and represented by Family Dependency factor (FDF) .FDF (member) $=1$ if the member is the source of income and FDF (member) $=0$ if he is not so. In most of the families specially in India GrandFather and Grand Mother will also live along with their children (even though nuclear families are becoming more popular nowadays). In such families preference and special respect will be give to such senior citizens. This relationship is quantified in terms of Age Factor (AF) which is computed based on their age as follows:

$\operatorname{Af}(x)=\left\{\begin{array}{cc}1 & \text { if } 50<x<100 \\ \frac{\operatorname{age}(x)-10}{40} & \text { if } 10<x<50 \\ 0 & \text { otherwise }\end{array}\right.$
Social Dominance factor for a given member is computed using equation (4)

$\mathrm{SDF}\left(\mathrm{U}_{\mathrm{i}}\right)=($ Role Factor + FamilyDependency Factor + AgeFactor)/3 ---(4)

User participation is also important while considering the preferences. If the user has participated very less time in this social activity he has to be considered with more weightage. The participation factor is computed using equation (5) .(pt stands for participation time, $\mathrm{tt}$ - represents total viewing time).

$$
\operatorname{PaF}\left(\mathrm{u}_{\mathrm{i}}\right)=1-\frac{p t}{t t}
$$

The SocialPreferenceScore (SPS) for each person is computed considering the average of SDF and $\mathrm{PaF}$ as given below:

$\left.\operatorname{SPS}\left(u_{i}\right)=1 / 2\left(\operatorname{SDF}\left(u_{i}\right)\right)+\operatorname{PaF}\left(u_{i}\right)\right)$

Table1 illustrate one of the families with their social status, relationship and dominance (importance).

Table1:Social Relationships and dominance in a given Father dominant family

\begin{tabular}{|c|c|c|c|c|c|c|c|c|}
\hline Sl.No & Role & Age & RF & FDF & AF & SDF $\left(\mathbf{U}_{\mathbf{i}}\right)$ & PaF $\left(\mathbf{u}_{\mathbf{i}}\right)$ & SPS $\left(\mathbf{u}_{\mathbf{i}}\right)$ \\
\hline U1 & Father & 40 & 1 & 1 & 0.75 & 0.91666 & 0.8 & 0.5583 \\
\hline U2 & Mother & 35 & 1 & 0 & 0.625 & 0.51566 & 0.2 & 0.35783 \\
\hline U3 & Son & 12 & 0.5 & 0 & 0.05 & 0.18333 & 0.7 & 0.44167 \\
\hline U4 & Daughter & 07 & 0.5 & 0 & 0 & 0.16666 & 0.5 & 0.3330 \\
\hline
\end{tabular}

In real-time situations user preferences are truly influenced by the member surrounded by him at that moment of watching TV. Even though his /her personal choice is different many times user will compromise to the group preference .Sometimes because of group mood / behavior the person may get motivated to different mood and preferences. As a result the members individual ratings also changes whenever they are in the group. Preference Score of any member for a given service (PS) can be expressed in terms of personal preference and social influence
(SPF). Equation 7 gives the relationship between user and family (group) influence.

$$
\operatorname{PS}_{\mathrm{ui}}(\mathrm{Si})=\mathrm{UR}_{\mathrm{ui}}(\mathrm{Si}) * \operatorname{SPF}\left(\mathrm{u}_{\mathrm{i}}\right)
$$

Table 2 illustrate the influence of Social Preference scores on their personal preference scores . 
Table 2: Influence of Group on individual

\begin{tabular}{|l|l|l|l|l|l|l|l|l|l|}
\hline User & Role & Age & SPS $\left(\mathrm{u}_{\mathrm{i}}\right)$ & $\mathrm{UR}(\mathrm{P} 1)$ & $\mathrm{UR}(\mathrm{P} 2)$ & $\mathrm{UR}(\mathrm{P} 3)$ & $\mathrm{PS}(\mathrm{P} 1)$ & $\mathrm{PS}(\mathrm{P} 2)$ & $\mathrm{PS}(\mathrm{P} 3)$ \\
\hline Raj & Father & 40 & 0.5583 & 50 & 40 & 10 & 27.91 & 22.332 & 5.583 \\
\hline Sheela & Mother & 35 & 0.35783 & 20 & 50 & 30 & 7.1566 & 17.89 & 10.73490 \\
\hline Vinayak & Son & 12 & 0.44167 & 30 & 30 & 40 & 13.25 & 13.25 & 17.6668 \\
\hline Palguni & Daughter & 07 & 0.3330 & 40 & 40 & 20 & 13.32 & 13.32 & 6.666 \\
\hline
\end{tabular}

\section{P1, P2, P3 are programs, UR is the respective user ratings, PS is the preference scores, SPS -Social Preference Score}

Ultimately the goal of the system is to satisfy all users. The satisfaction is measured implicitly using equation (8) and explicitly through the feedback obtained from the user. The Satisfaction Value (St) is Computed using equation:

$$
S t=1-(\mathrm{G} / 100)-------(8)
$$

Where $\boldsymbol{G}=\boldsymbol{d}$ (User Highest Preferred Service, User Preference of Group Preferred Program)

\subsection{Context Modeling:}

Context Modeling is a fundamental step for every adaptation application and the development of context aware systems .Context modeling addresses the issue of how to represent the contextual information. Schilit in his PhD thesis used a simple model with context being maintained by a set of environment variable. Several approaches were proposed for the representation of context. In Policy based context model all context information will be mapped into the policies. Policy takes the following rule base format If $\{$ condition(s) $\}$ Then $\{\operatorname{action}(\mathrm{S})\}$.For Example If(Location $==$ meeting Room $)$ and (time within meeting Schedule )Then Mobile Vibrate Only.

A survey made by Strang eta al contains an interesting comparative study of different modeling methods .To conclude the ontology makes the best description of context compared to the surveyed methods because it provided a good sharing of information with common semantics .Even though the ontology seems to be the most suitable tool for context modeling ontology tools are only good at semantically representing the knowledge of a domain .They are not designed for capturing and processing constantly changing information in dynamic environments in a scalable manner. Moreover existing ontology languages and serialization formats are text based and therefore are not designed for efficient query optimization, processing and retrieval of large context data. The proposed system makes use of hybrid ontology and fuzzy logic to manage context semantics and relational approach to manage context data. Ontology is a set of structured concepts that are organized in a graph where relations can be either semantic relations or composition and heritage relations. The main objective of ontology is to model the set of knowledge of a given domain. This means to choose a manner for describing domain information in a form comprehensible by computers. [35, 36, 37, 38, 39, 40, 41, 42]

Since Bayesian networks require discrete data, they generally have disadvantages though they are poising tools for reasoning context. They cannot deal with various types of information effectively because discretization can lose information compared with the original one .Context inference module can use several types of sensor information. Some are continuous and other are discrete .Besides it is possible for one data to be categorized into several states at the same time .Usually a state with the largest value is selected as its state, but this method has a problem when the value is near the criteria or the value belongs to several categories. We have used the fuzzy system for pre - processing step for Bayesian network inference since the fuzzy system is relevant in dealing with diverse information and uncertainty.

Context is used to represent the family members situation with respect to watching TV. It is a vector of vectors. The values of context parameters are either discrete or continues. The raw contextual data is preprocessed so that they are represented as a fuzzy membership vector. The fuzzy membership functions may be singleton, trapezoidal and triangular functions $[43,44,45]$. In our proposed work context is set of fuzzified instances of primitive context like location, time, userid, activity and mood. The fuzzy membership functions used in our proposed work is singleton and is defined as follows :

\section{$f($ Context $)= \begin{cases}1 & \text { if Context } \in A \\ 0 & \text { if Context } \notin A\end{cases}$}

Where A is the set of values with fuzzy boundaries. Table 3 lists out some of the fuzzy values for a given context.TV will be monitoring continuously the context of its surroundings particularly that of users. Depending on the context change it will provide the appropriate services.Fig4, Fig5 and Fig6 illustrates some of the situations in the Living Room.

Whenever the new context is detected the system compares that with the existing current context using distance formula. Dalal(1988) proposes a distance concept for belief revisit. We adopt Dalal's distance concept to measure the inconsistency between two contexts.

Table3: Fuzzy Values for Example Sets

\begin{tabular}{|l|l|}
\hline Context & \multicolumn{1}{|c|}{ SET } \\
\hline Location & $\begin{array}{l}\text { SofaSet, Chair1, Chair2, Floor, Table1, Table2, } \\
\text { Entrance, DiningTable. }\end{array}$ \\
\hline Time & $\begin{array}{l}\text { AM1, AM2, ------AM12, PM1------PM12, Early } \\
\text { Morning, Morning, Afternoon, Forenoon, Evening, } \\
\text { Night, Late Night. }\end{array}$ \\
\hline Activity & $\begin{array}{l}\text { Standing ,Sitting, Running ,Walking , Jogging, } \\
\text { Eating , Talking ,Resting ,Sleeping ,Playing } \\
\text {,Reading,Exercise, , }\end{array}$ \\
\hline Mood & $\begin{array}{l}\text { Happy,Normal,Relax,Cheerful,Deppressed,Excited, } \\
\text { Disturbed,Comfort,Angry, Disturbed }\end{array}$ \\
\hline
\end{tabular}




\begin{tabular}{|l|l|l|l|l|}
\hline User situation & Role & Activity & Mood & TV S TATE \\
\hline & Father & Sitting on sofa & Homal & \\
\hline & Mother & Washing Floor & Homal & \\
\hline & Son & Crying & Sad & Switched OFF \\
\hline
\end{tabular}

Fig4: Users situations and TV Action



Fig5: Favorable Postures and activities to watch TV

Distance between two contexts $\mathrm{Ci}$ and $\mathrm{Cj}, \mathrm{Dc}(\mathrm{Ci}, \mathrm{Cj})$ is given by $\operatorname{Dc}(\mathrm{Ci}, \mathrm{Cj})=\mathrm{D}(\mathrm{Vi}, \mathrm{Vj})=\sum_{k=1}^{k=n} d(a k, b k)$

whereas ak \&Vi and bkeVj.

$\mathrm{Vi}$ and $\mathrm{Vj}$ are corresponding vectors extracted from $\mathrm{Ci}$ and $\mathrm{Cj}$.

Table4: Context C1

\begin{tabular}{|l|l|l|l|l|}
\hline Location & Time & Userid & Activity & Mood \\
\hline Chair1 & AM1 & $\# 1$ & Sitting & Normal \\
\hline Chair2 & AM1 & $\# 2$ & Sitting & Normal \\
\hline Sofa & AM1 & $\# 3$ & Sitting & Normal \\
\hline Sofa & AM1 & $\# 4$ & Sitting & Normal \\
\hline
\end{tabular}

ContextChange $(\mathrm{Ci}, \mathrm{Cj})=\left\{\begin{array}{l}y e s \text { if } D c(C i, C j) \geq 1 \\ N o \text { if } D c(C i, C j)=0\end{array}\right.$

Few of the events which results in change in the context to list are

- $\quad$ Change in the number of family members watching TV

- $\quad$ Change in the group/user mood .

- Change in the group/user activity.

- Change in the time

- Change in the program in that respective channel,etc.,



Fig6: Postures and activities which are not favorable to watch TV

Context Representation: Each context is represented as Relational Table with name of the table representing the context id (ex $\mathrm{C} 1, \mathrm{C} 2,----)$, columns specifying primitive contexts instances like (userid,etc).Each tuple representing the context of single user. Multiuser context will be represented using multiple tuples. The Maximum number of tuples in a table depends on the number of family members participated in the context.Table4 illustrate one such context representing four family members inside the living room.

The context are stored using index database as shown in the figure7 . c1,c2,c3,----are context ids which are pointing to respective context tables.

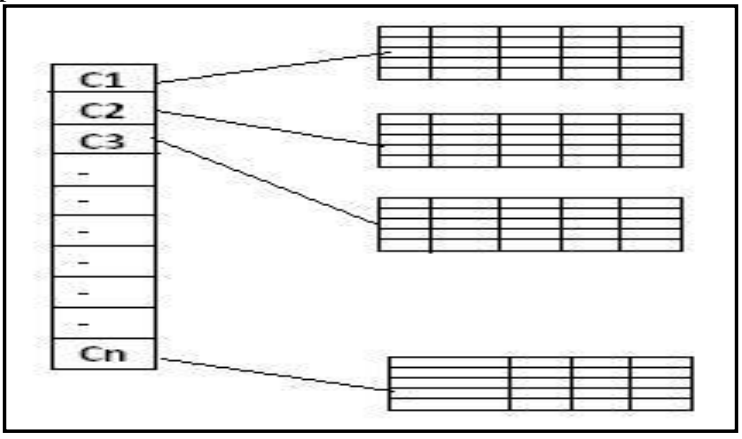

Fig7: Context Repository 


\section{3) TV Domain:}

TV is modeled in terms of its programs and settings .The Contents of TV Service can be classified into Categories, Channel and Program .User usually prefers channel based on its quality and program. For experiment we are considered few categories of TV programs. In each program member will have his own likings and dislikings. Table5 gives a few list of the categories with example set and probable user interesting content..

While computing reference scores the recommendation engine has to take into account the channel quality, users liking and disliking contents in the program. The user liking factor (ULF) is defined as

$$
\mathrm{ULF}=\left\{\begin{array}{l}
1 \text { if }|p i f-p d f|>\varepsilon \\
0 \text { if } \mid \text { if }-p d f \mid \leq \varepsilon
\end{array}\right.
$$

Whereas

$\operatorname{pif}($ ui $\mid \mathrm{p})=\frac{1}{|n i f|} \sum_{j=1}^{j=n} l f j(u i \mid p)$

$/ /$ pif - program interesting factor

$\operatorname{pdf}($ ui $\mid \mathrm{p})=\frac{1}{|n d f|} \sum_{j=1}^{j=n} d l f j(u i \mid p)$

$/ / \mathrm{pdf}$ - program disliking factor

lf - liking factor dlf-disliking factor $\varepsilon$ is experimentally determined and its value is 0.4 .

Table5: Categories with user interest contents

\begin{tabular}{|c|c|c|}
\hline Categories & Subcategories & $\begin{array}{c}\text { Users Interesting } \\
\text { Content }\end{array}$ \\
\hline Music & $\begin{array}{l}\text { Classical, Rock, Soft } \\
\text { Music, Pop Songs, } \\
\text { Album Songs, New } \\
\text { Releases, Jackson, } \\
\text { Folk Songs ,etc } \\
\end{array}$ & $\begin{array}{l}\text { Artist, Album, Music } \\
\text { Director, Team, Live }\end{array}$ \\
\hline Sports & $\begin{array}{l}\text { Tennis, Cricket, } \\
\text { Foot Ball, Common } \\
\text { Wealth, Olympics, } \\
\text { World Cup, }\end{array}$ & $\begin{array}{l}\text { Live, Players ,Country } \\
\text { playing,Live Match }\end{array}$ \\
\hline Movies & $\begin{array}{l}\text { English Movies } \\
\text {,Hindi Movies , } \\
\text { Regional Language } \\
\text { Movies }\end{array}$ & $\begin{array}{l}\text { Action, Detective, } \\
\text { Thrill ,Suspense ,Love } \\
\text {,Technical ,Actors } \\
\text {,Director, Latest ,Old, } \\
\text { Devotional .Comedy }\end{array}$ \\
\hline News & $\begin{array}{l}\text { English News, Hindi } \\
\text { News, Regional } \\
\text { Language News, } \\
\text { National } \\
\text {,International , } \\
\text { Business News, } \\
\text { Political News, } \\
\text { Cinema News, } \\
\text { Election News, }\end{array}$ & $\begin{array}{l}\text { National ,International, } \\
\text { Regional ,Local, } \\
\text { Politics, Cinema, } \\
\text { Sports , Breaking, etc }\end{array}$ \\
\hline Cartoon & $\begin{array}{l}\text { Tomm and Jerry, } \\
\text { Chota Bheem, Micky } \\
\text { and Donald, etc }\end{array}$ & New Series, Old Series \\
\hline Education & Science, Gadgets, & Breakthrough \\
\hline
\end{tabular}

Based on the user's degree of interest and current context the preference score for the program is computed using equation (13).

\section{PreferenceScore $($ TVProgram/Context $)=$ $\sum_{\forall C a} P(T V P r o g r a m \mid C a) X$ ULFui(TvProgram)}

Where $\mathrm{P}(\mathrm{TV}$ program $/ \mathrm{Ca})$ is computed using Bayesian probability. $\mathrm{Ca}$ is vector of context attributes (mood, day and time).Many times a single program will be played by multiple channels .In such case the channel will be selected based on its quality features.

$$
\begin{aligned}
& \operatorname{Ch}(q f)=\frac{1}{|n q f|} \sum_{j=1}^{j=n} q f i(\text { ch }) \\
& \text { PreferenceScore }(\text { Channel })=\left\{\begin{array}{l}
1 \text { if } \operatorname{Ch}(q f)>\epsilon \\
0 \text { if } \operatorname{Ch}(q f) \leq \varepsilon
\end{array}\right.
\end{aligned}
$$

$$
\text { Capreference }=\sum_{\forall C a} P(\text { Category } \mid \text { Context })
$$

\section{PreferenceScore $($ Category $\mid$ Context $)=$ (1) if Capreference $>0$ $\{$ if Capreference $\leq \varepsilon$}

Similar calculations have been done for TV Settings .

Recommendation process: The working of proposed recommendation engine for TV is shown in Figure8.

Recommendation engine recommends suitable Channel and settings making use of any one of two approaches based on the current context

1. Query Processing Approach: If the current context matches with any one of the context present in the context repository then the context id will be retrieved with the help of SQL query. Based on the retrieved context id the corresponding actions will be recommended using actions database. MySQL Database is used to serve the purpose. 




Fig8 : Architecture of the proposed system

2. Algorithmic Approach : If the current context is a new context and does not matches with any of stored context. Recommender stores a context in the context repository and assigns a new id. The set of actions for new context will be assigned by using single and multiuser preference estimation algorithm described in the section IV. The algorithms are designed using Bayesian Networks using fuzzy evidence as illustrated in fig9. The recommendation makes use of Bayesian theorem to predict the appropriate service in a given context i.e

$\mathrm{P}($ Service/Context $)=\frac{\mathrm{P}\left(\frac{\text { (context }}{\text { Service }}\right) \times \mathrm{P}(\text { service })}{\mathrm{P}(\text { Context })}$

The service with highest probability will get recommended. The Service may be TV Channel, TV Program, Volume, Brightness, etc. Context may be single user or multiuser context (including

\section{PROPOSED ALGORITHM}

In this paper we are proposing new Algorithm for Estimating User Preferences in a single user and multiuser context. The algorithm is an improvement of our earlier work $[46,47,48,49]$. There have been studies to combine the fuzzy system and Bayesian networks .Yang proposed fuzzy Bayesian approach that estimates the density function from the conditional probabilities of the fuzzy supported values in order to use continuous value in Bayesian framework. Pan and Liu proposed fuzzy Bayesian network and inference algorithm using virtual nodes and Gaussian functions. However these methods have constraints where observed information should be only one and membership degree sum should be one Our proposed model includes simple and effective fuzzy Bayesian network without those constraints. location, mood, activity and time).Usually the context does not grow abruptly after certain days . Context cache is used to store the frequently occurring context

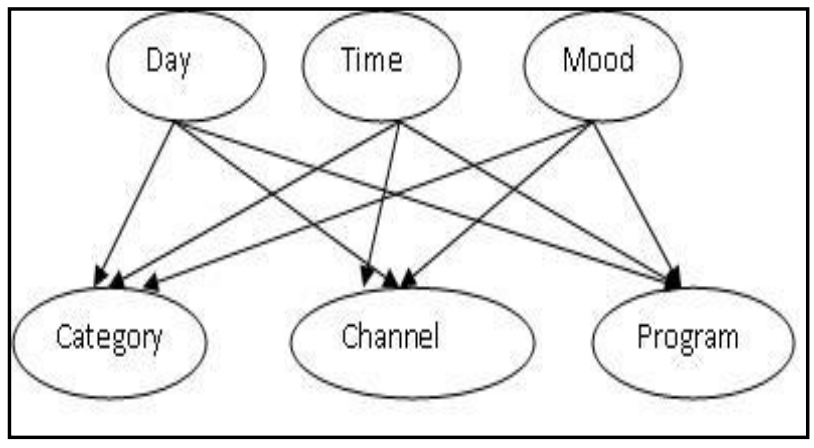

Fig9: Bayesian Network

Algorithms make use of Bayesian Classifier to predict the user using context repository, action repository, user profile and internet. Algorithm is used to play TV automatically whenever the appropriate context is detected (single user context or multiuser context) .

Prediction of the Single user preference channel and settings:

The user preference is predicted by computing the Recommendation score for service like Program and settings.

S1: RS((TVProgram, Ch, Category $) /$ Context $)=$ PS(Category)*PS(Ch)*PS(Program)

- $\quad[\mathrm{RS}-$ Recommendation Score PS - Preference Score] 
- $\quad$ PS(Category),PS(Ch), and PS(Program) are computed using equations 13,15 and 17 respectively.

S2: UserPrefered $(\mathrm{Ch} \mid$ context $)=$ ch with Max

\{RecommendationScore ((TVProgram, Ch, Category)/Context) UserPrefered (Settings $\mid$ context $)=$ Settings with Max $\{$ RecommendationScore ((Volume, Brightness , Color) $\mid$ Context ) \};

Estimating Group Preferred Channel and Group Preferred Settings using fuzzy Bayesian collaborating filtering method

When the no of users are more than one collaborative filtering has to be done for recommendation .Preferences of the user changes in a group based on their role and age in the family. The family we have considered in this paper is father dominant nuclear family. The Group preference (GP) of user ui for required service will be calculated using equation 19 as follows:

\section{GPui $(($ Service $) /$ Context $))=$ UPui $($ Service $\mid$ Context $) *$ $\left(\operatorname{SPF}\left(\mathbf{u}_{\mathbf{i}}\right)\right) ;---\cdot---(19)$}

Consider a scenario where in Father, Mother ,Son and Daughter are watching TV at certain Time in Living room.

In such case the group preferred channel is calculated as follows

$$
\begin{aligned}
& \mathbf{G P}_{\text {Father }}=\Pi\left(\mathbf{U P}_{\text {Father }}(\mathrm{Ch} \mid \text { Context }), \mathrm{SPF}_{\text {Father }}\right) \\
& \mathbf{G P}_{\text {Mother }}=\Pi\left(\mathbf{U P}_{\text {Mother }}(\mathrm{Ch} \mid \text { Context }), \mathbf{S P F}_{\text {Mother }}\right) \\
& \text { GP }_{\text {Son }}=\Pi\left(\mathbf{U P}_{\text {Son }}(\mathrm{Ch} \mid \text { Context }), \text { SPF }_{\text {Son }}\right) \\
& \text { GP }_{\text {Daughter }}=\Pi\left(\mathbf{U P}_{\text {Daughter }}(\mathrm{Ch} \mid \mathrm{Context}), \mathrm{SPF} \text { Daughter }\right)
\end{aligned}
$$

\begin{tabular}{|c|c|c|c|c|c|c|c|}
\hline User & uGP(il) & uGP(P) & uGP(i3) & uGP(i4) & - & - & uGP(in) \\
\hline F & Rfil & Rfi2 & Rfi3 & Rfi4 & & & Rfin \\
\hline$M$ & Rmil & Rmi2 & Rmi3 & Rmi4 & - & - & Rmin \\
\hline 5 & Ril & Rsi2 & $\mathrm{Rs} 3$ & $\mathrm{Rsi4}$ & - & - & Rsin \\
\hline D & Rdil & $\mathrm{Rdi2}$ & $\mathrm{Rdi3}$ & Rdi4 & . & - & Rdin \\
\hline AR & Hil & $\mu 2$ & $\mu 3$ & ii4 & & & min \\
\hline
\end{tabular}

Table6: Family members and their social preference in a group.

To measure the similarity between the user's interests in a family we have used Pearson algorithm. The algorithm is is based on the use of sandard Pearson $r$ correlation coefficient. The possible values of the Pearson coefficient range from -1 to +1 including 0 . Values near -1 indicate a negative correlation while values close to +1 indicates a positive correlation; a value of 0 shows no correlation at all. Once the Pearson coefficient has been calculated the recommendation can be done by averaging the values of the most similar profiles .One important characteristic of this it takes into account not only positive correlation but also negative correlation to make the predictions .The formula used to calculate the similarity between users takes into account the rates of songs provided by both users and it is defined below .

$$
\mathbf{D}_{\mathbf{x y}}=\frac{\sum_{m}(P x m-\mu x)(P y m-\mu y)}{\sqrt{\sum_{m}(P x m-\mu x)^{2} X \sum_{m}(P x m-\mu x)^{2}}}
$$

Where Pxm is the rate of program given by user $x$ Pym is the rate of program $m$ given by the user $y$. $\mu \mathrm{x}$ is the average rate of user $\mathrm{x}$ $\mu y$ is the average rate of user $y$.

The formula (20) defines the way similarities between users are measured .This formula takes into account only programs that were rated by both users and measures the difference between each common program and the average of the rates of each user Once all the correlations between users are calculated then the prediction of a specific program can be calculated easily as a weighted average of all the user ratings for this program with the formula

$$
P x i=\mu x+\sigma x \frac{\sum_{k}^{N u} D k x\left(\frac{P k i-\mu \mathrm{k}}{\sigma x}\right)}{\sum_{k}^{N u} D k x}
$$

Where Pxi is the predicted rate for user $\mathrm{x}$ on item $\mathrm{i}$ Pki is the rate of program I for given by user $\mathrm{k}$ Dkx is the correlation between user $\mathrm{k}$ and user $\mathrm{x}$ $\mu \mathrm{x}$ is the average rate of user $\mathrm{x}$ $\sigma \mathrm{x}$ is the standard deviation of all the rates of user $\mathrm{x}$.

Fig10 gives the algorithm designed for the proposed work.Fig11 illustrates one of the possible outcome of the algorithm. 


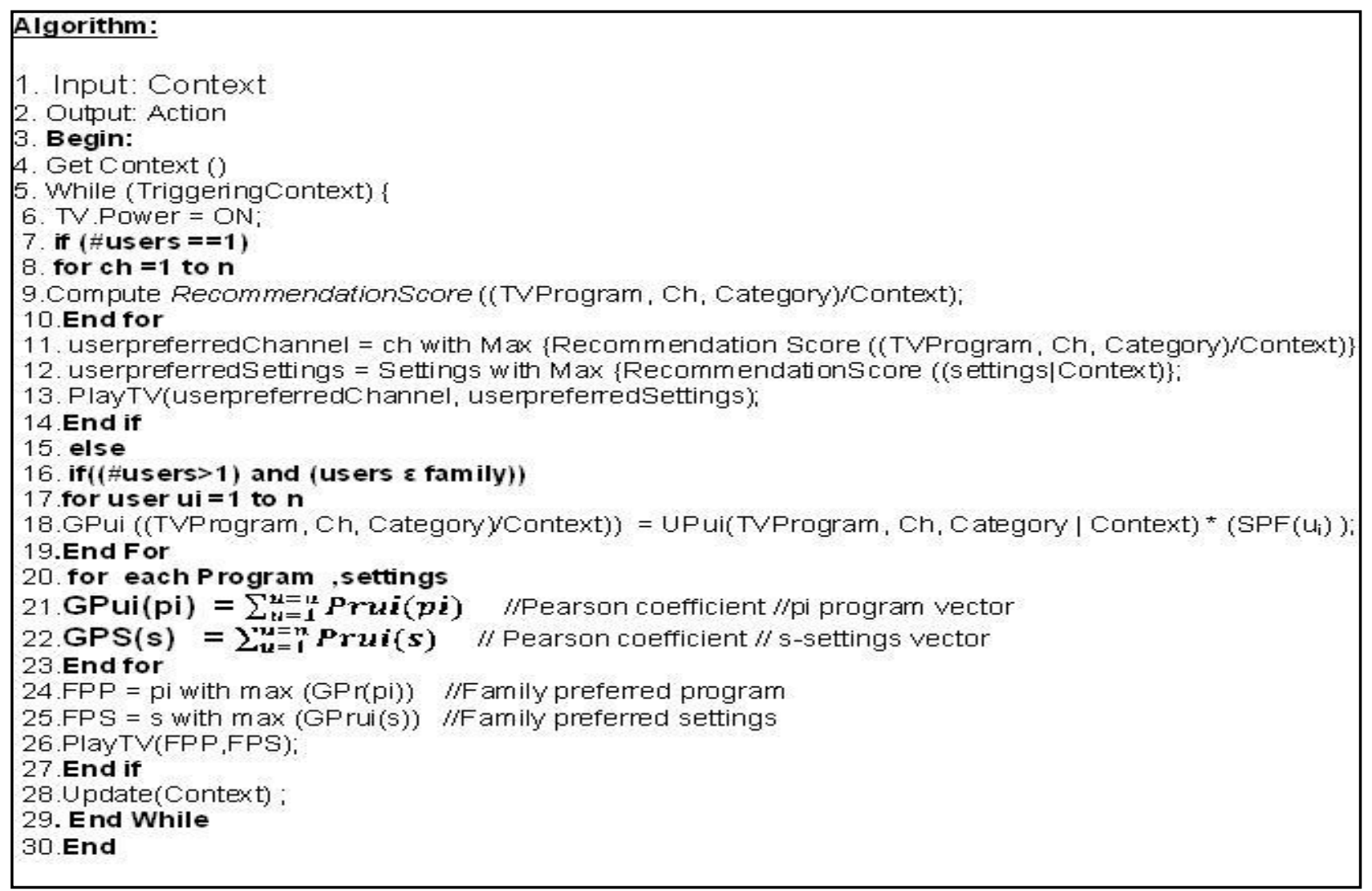

Fig10 : Algorithm to recommend tv program and settings

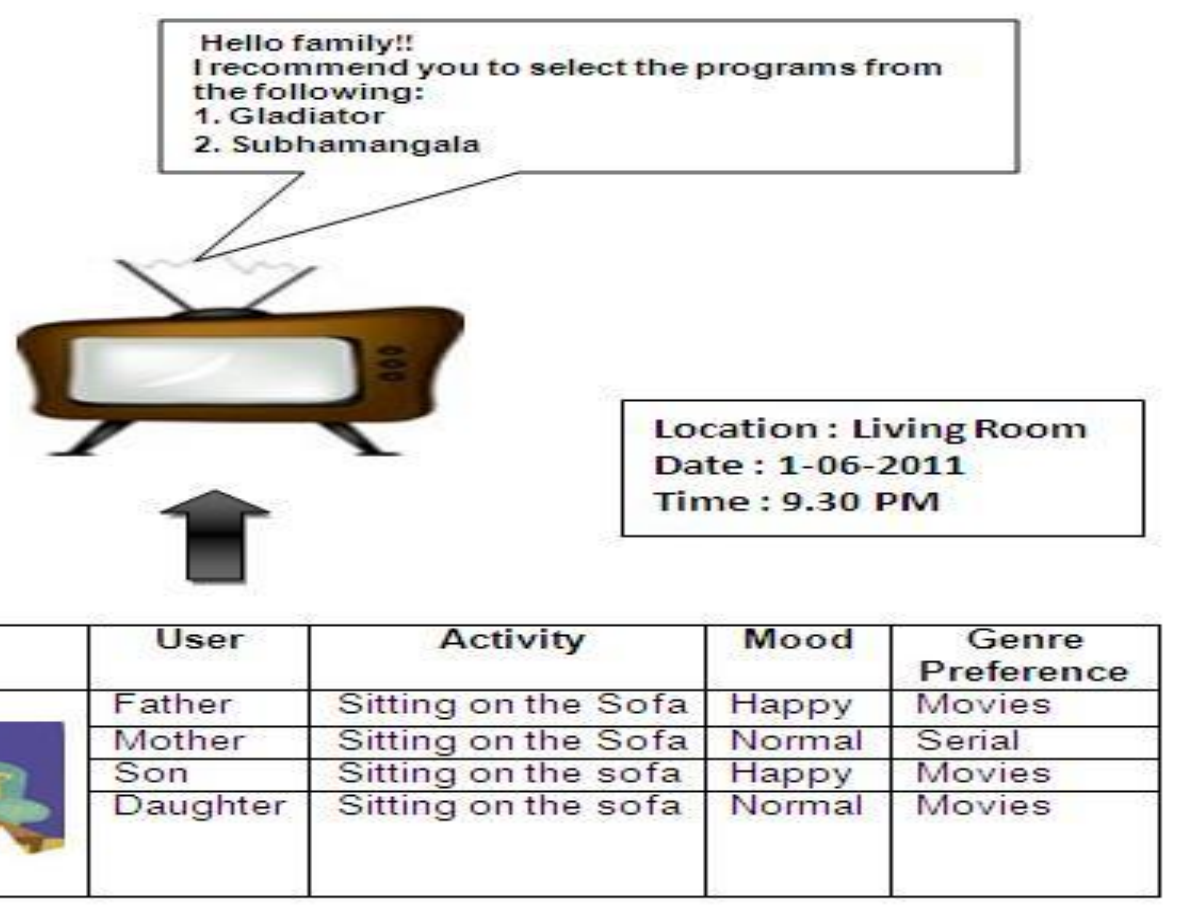

Fig11 : Recommendation of service to the family 


\section{IMPLEMENTATION}

We implemented the proposed recommendation system making use of the simulated TV. A survey report of about 100 popular Indian Channels of different category was used for simulations. Activities and Mood was collected through interviewing (35 Small Indian Families) and observing closely the tv watching pattern of the three small families comprising Father, Mother, Son and Daughter (For about Six Months) (For details see Appendix).

The implementation of the proposed system was done in three phases:

1. DataCollection (Database Design)

2.Implementation of Context Aware Recommendation Engine

3. Development of Graphical User Interface

\subsection{Data collection}

\subsubsection{Family Data}

Collection of data is the foundation of any personalized service .Personalization is achieved through explicitly asking user ratings and implicitly collecting related observations where the latter approach is usually more important. Initially all users has to get register with system by entering their profiles (name, role, age, likings and disliking) and ratings under different context. The generated context is matched with the stored Context Cache (or Context history) if found the respective actions will be triggered. Otherwise if the generated context is a new context Recommendation engine will estimate a situation based service using watching history and proposed algorithms and the new context and respective actions (generated) will be stored..Using Users Watching History Database two new databases will be created called context history and action history. Recommendation scores for a given situation will be computed only when the new context arises. However the Context Cache and Action Repository will be update regularly for every one month (30 days) based on users watching history.

If a user previously liked a program in a given situation the same program and other similar programs can be expected to be good recommendations when the same user and situation are encountered the next time. Respectively, if the user disliked or skipped a program, it should not be recommended again. When the user watch more than one program in a given fuzzy time in such case the program or channel viewed long time (or interested to user obtained through interaction ) will considered and stored in the Watching History through data filtering.

\subsubsection{Context data}

The present work is based on simulator where in the user explicitly describes his situation. User-provided situation data are directly applicable for program recommendation. Considering tv, one very important piece of context is the emotional state of the viewer - after all, tv program is intrinsically communication about emotions. In practical systems, the emotions or moods of the user cannot be directly sensed, but they can for example be asked. Also, it is important to realize that when program is played according to its mood, we can perhaps glean information about the mental state through the watching history, which we already are collecting. The accelerometer is sufficient to recognizing movement and activity to some degree. For example, standing still, walking, running, or vehicular movement can be recognized from each other by the accelerometer signals. Activity can also be observed from the phone usage data, starting with simple phone profile information (general, silent, meeting, etc.).

\subsubsection{TV program data}

TV program recommender systems also harvest programs metadata in addition to collecting user and context data. Such metadata contains textual titles of the genres, channel, time, program name, program id, etc., The metadata is needed to be able to associate different pieces of music with each other, and to help alleviate the sparsity of the rating and listening data.

\subsection{Implementation of Context Aware Recommendation Engine}

The heart of the personalized TV service is the program purpose model, which encompasses information about all programs viewed in the service by all users in all situations. The purpose of the program purpose model is to make predictions, given observations of user, tv program content, and context features. Predominantly the predictions are context-aware tv program recommendations, i.e., suggestions on tv program to be watched in the user's current situation. The proposed recommendation engine is based on hybrid of fuzzy Bayesian Classifier and Collaborative filtering.For Individual user preferences Fuzzy Bayesian Classifier is used and for Group preference statistical methods based collaborative filtering is used. The recommendation system is implemented using C\#.NET and MySQL database.

\subsection{Graphical User Interface:}

Fig12 below shows the User Interface of the simulator .While simulating one can create a context by selecting appropriate values for Location, Role, Activity and Mood. The values for Channel ,Volume, Brightness and Color will be computed by the system using our proposed algorithms making use of user profile and history database. When the Start Simulation button is pressed TV starts to play with the appropriate channel. When the new context is created different users and their satisfaction level will also displayed. Fig12 show one such instance wherein four users with different mood are watching TV .Refresh history button is provided to refresh the history if needed. 


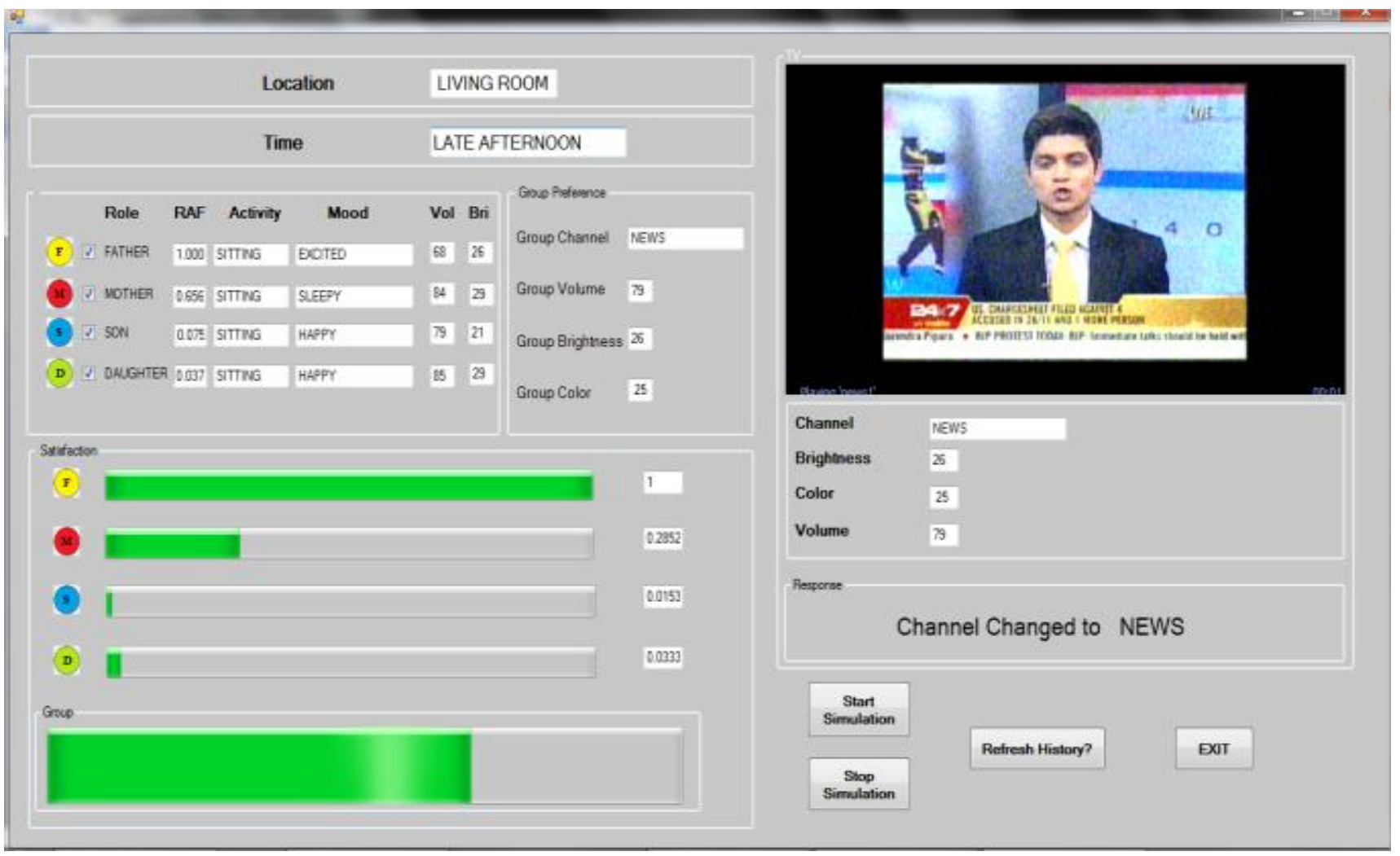

Fig 12: GUI of Simulated Context Aware TV

\section{EXPERIMENTAL DATA AND RESULT ANALYSIS}

Totally 35 family were interviewed for survey report and three family (with different tv watching pattern) were invited to test the system The recommendation system was developed using C\# .NET (Microsoft Visual Studio 2005). The experiments involved a total of 100 live channels with different genres like serial, movies, cartoon, music, sports, advertisements, etc. The six months TV watching pattern history of three families was collected through daily questionnaires and observations. The data base was stored in the respective database. The family members were made to registered their profile initially. They are also made to enter the current context (location, mood, activity and preferences). We performed four experiments to test the recommendation accuracy, performance and users satisfaction level.

Experiment 1: In this experiment, we aimed to evaluate the Precision of recommending the appropriate TV programs based on the current context of the family members. The average precision was calculated for every week . The recommendation precision was observed for entire 30 weeks .It was evaluated for three families. Table7 illustrates the watching pattern of three different families.
Table7: Family TV Watching patterns.

\begin{tabular}{|l|l|l|}
\hline Family & Frequency & $\begin{array}{l}\text { Watching } \\
\text { TVTime }\end{array}$ \\
\hline Family1 & Most Frequently & 6-14hrs per day \\
\hline Family2 & Less Frequently & 2-6hr per day \\
\hline Famly3 & Highly irregular & 0-14hrs per day \\
\hline
\end{tabular}

Fig13 shows the variation of precision wrt to week numbers. For all the families the recommendation accuracy increased smoothly .The system showed good performance for the family 1 which had consistent watching pattern. However in the week between 11 and 13 the system recommendation performance was decreased sharply because of the sudden change in the watching pattern (due to the world cup cricket).The maximum recommendation precision observed was $0.85,0.72$ and 0.43 for family 1 , family 2 and family 3 respectively. Because of the poor relationship with TV the system showed very less precision for the family3.

Precision $=\frac{N p}{T R p}$

$\mathrm{N}_{\mathrm{p}}$ is the number of programs family (member) actually watched in the respective week

$\mathrm{TR}_{\mathrm{p}}$ is the total number of recommended programs in the respective week. 




Fig13: Precision of different families

Experiment 2: In this experiment we aimed to evaluate the satisfaction levels of different users in different situations like watching tv individually and watching tv with the family members. Fig14, Fig15 and Fig16 illustrates the satisfaction levels of family members individually and as a group of Family1 ,Family2 and Family3 respectively.

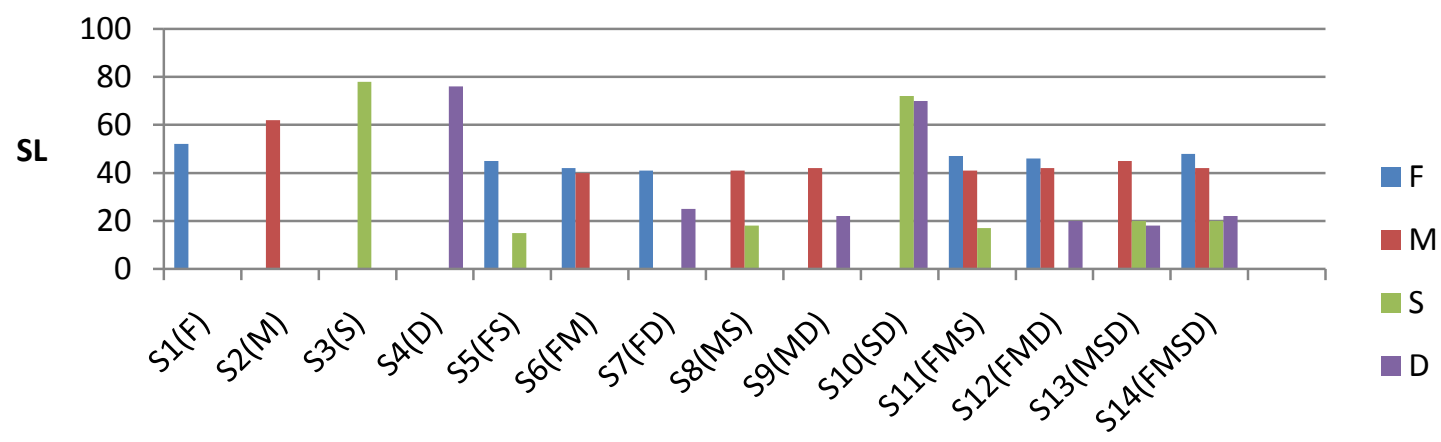

Family Situation

Fig14 : Satisfaction level(SL) of Family1in different situations

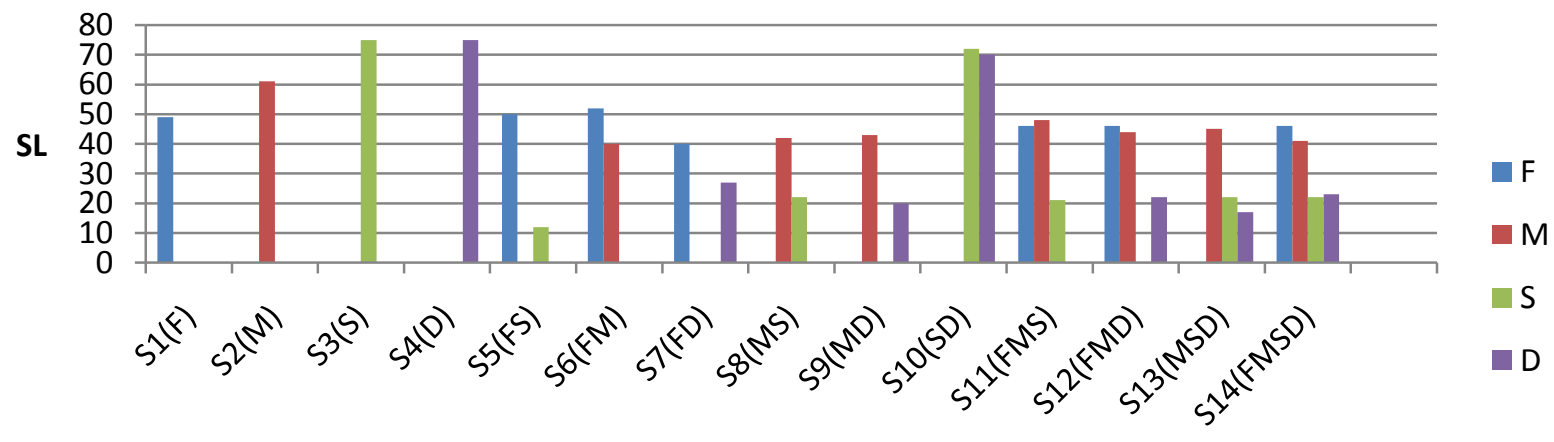

Family Situation

Fig15: Satisfaction level of Family2in different situations 


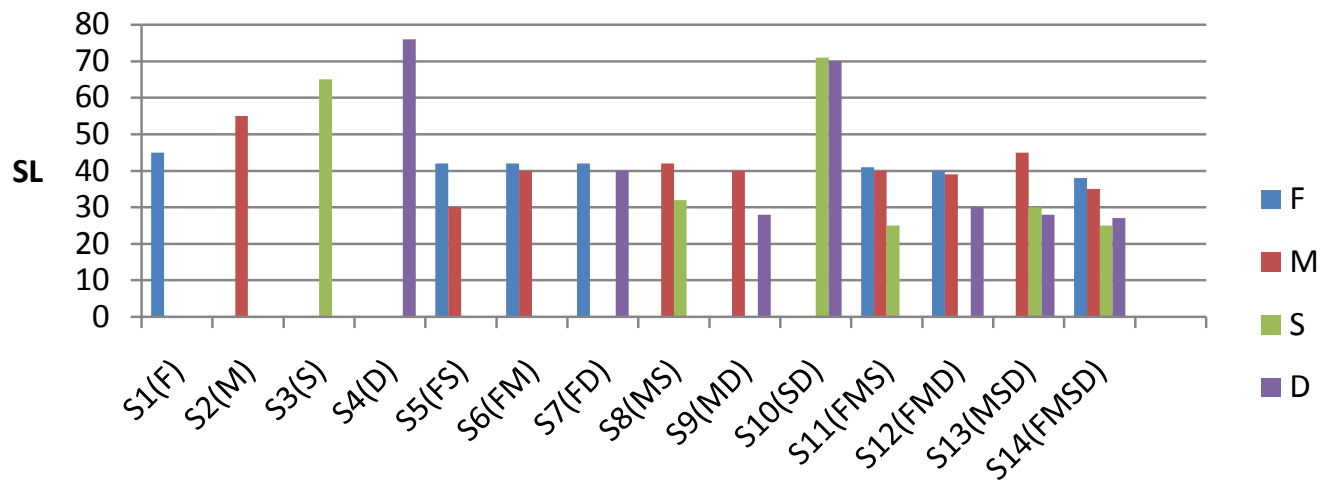

Family Situation

Fig16: Satisfaction level of Family3 in different situations

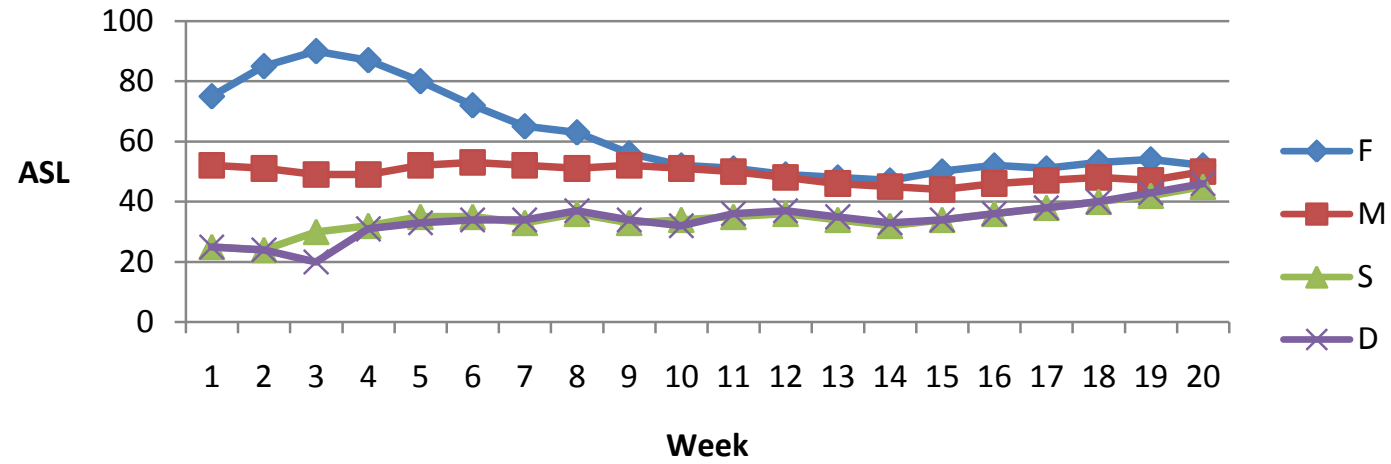

Fig17: Average Satisfaction level of F, M, S and D while watching TV together

The recommendation system even though initially it works in the favor of person with highest social dominant factor. But after certain amount of time period it works in the favor of entire family .Even though individual satisfaction level in a family decreases but it reduces the verbal conflict among the members. Fig17 shows the relation between time satisfaction level. The effect is due to the fuzzy Bayesian Network.

Experiment3: In this experiment we aimed to evaluate the

Table 8: Performance of Recommendation System

\begin{tabular}{|l|l|l|l|}
\hline Tasks & Time & Storage & $\begin{array}{l}\text { Memory } \\
\text { Size } \\
\text { required }\end{array}$ \\
\hline $\begin{array}{l}\text { Context } \\
\text { Searching and } \\
\text { Matching }\end{array}$ & $50 \mathrm{~ms}$ & User profiles & $320 \mathrm{~kb}$ \\
\hline $\begin{array}{l}\text { Service } \\
\text { Recommendation }\end{array}$ & $10 \mathrm{~ms}$ & $\begin{array}{l}\text { Look up user } \\
\text { profile }\end{array}$ & $100 \mathrm{~kb}$ \\
\hline $\begin{array}{l}\text { Individual } \\
\text { Preference } \\
\text { Calculation }\end{array}$ & $2 \mathrm{~s}-3 \mathrm{~s}$ & $\begin{array}{l}\text { Context } \\
\text { repository }\end{array}$ & $>1000 \mathrm{~kb}$ \\
\hline $\begin{array}{l}\text { Group } \\
\text { Preference } \\
\text { Calculation }\end{array}$ & $3 \mathrm{~s}-5 \mathrm{~s}$ & $\begin{array}{l}\text { Watching } \\
\text { history }\end{array}$ & $>5000 \mathrm{~kb}$ \\
\hline $\begin{array}{l}\text { Group Service } \\
\text { Recommendation }\end{array}$ & $1 \mathrm{~s}$ & TV Content & $500 \mathrm{~kb}$ \\
\hline Total & $\mathbf{6 . 0 6 s}$ to 9.06s & storage & $>\mathbf{6 9 2 0 k b}$ \\
\hline
\end{tabular}

performance of the recommendation system in terms of time and memory. The database used is MySQ1 .The time was measured using 1.73 GHZ CPU ,2GB RAM machine.

Experiment4: This experiment is survey based and it was aimed to understand the users watching pattern and to get the feedback on the proposed system. A survey of 35 small families comprising parents (Mother and Father) in the age of 35 to 45 and two children's in the age group of 5 to 15.The Family considered for survey was healthy Middle class families having single TV positioned at the living room. The family was run by Father or Mother or Both. The children were going to school and belonged to LKG to $10^{\text {th }}$ class. For collecting Feedback of the proposed system the system was made used by different families with different tv watching patterns. Fig16 and Fig17 gives the report of survey made. 
1.In your Family Who is More socially Dominant?

a. Father $-80 \%$ b. Mother- $20 \%$ c.Son $-0 \%$ d. $0 \%$

2. What categories of the programs are viewed when the entire family is present?

a. Sports ---5\% b.Entertainment (Movie/Music/Reality Shows/Serial) $---60 \%$ c.Cartoons $\quad--5 \%$ d.News $-28 \%$ f. Science and Education $-2 \%$

3. What categories of the programs are viewed when only parents are present?

a.Sports $---5 \%$ b. Entertainment (Movie/Music/Reality Shows/Serial) $\quad---65 \%$ c.Cartoons $\quad--1 \%$ d.News $-28 \%$ e. Science and Education $-1 \%$

4. What categories of the programs are viewed when only children's are present?

a. Sports ---1\% b.Entertainment (Movie/Music/Reality Shows/Serial) $---2 \%$ c.Cartoons $--95 \%$ d.News $-1 \%$ e. Science and Education $-1 \%$

5. What categories of the program mother wishes to watch when she is alone?

a.Sports $---1 \%$ b.Entertainment (Movie/Music/Reality Shows/Serial) $\quad---77 \%$ c.Cartoons $\quad-1 \%$ d.News $-20 \%$ e. Science and Education - $1 \%$

6. What categories of the program Father wishes to watch when he is alone?

a.Sports ---25\% b.Entertainment (Movie/Music/Reality Shows/Serial) $---40 \%$ c.Cartoons $\quad--1 \%$ d.News $-33 \%$ e. Science and Education $-1 \%$

7. What categories of the programs are viewed by the children's in the age group of 5 to 10 years?

a. Sports $--1 \%$ b.Entertainment (Movie/Music/Reality Shows/Serial) $---20 \%$ c.Cartoons $\quad--78 \%$ d.News $-0 \%$ e. Science and Education $-1 \%$

8. What categories of the programs are disliked by parents?

a.Sports ---10\% b.Entertainment (Movie/Music/Reality Shows/Serial) $---1 \%$ c.Cartoons $\quad--70 \%$ d.News $-5 \%$ e. Science and Education - 10\%

9. What categories of the programs are disliked by childrens?

a. Entertainment (Movie/Music/Reality Shows/Serial) $---20 \%$ b.Cartoons $\quad--0 \%$ c. Sports/ News /Science and Education $80 \%$

10. What categories of the programs are prevented to children's?

a.Adult Movies $-100 \%$

11. How often your family verbally fight over TV programs?

a. A lot $5 \%$ b. Sometimes $20 \%$ c.Occasionally $74 \%$ d. Nill $1 \%$

\section{Fig18 : Questions Asked with Persons related to TV watching pattern and program}

1. Did the recommendation help you to discuss the program options? a.Positive Answer $-85 \%$ b.Negative Answer -10\% c.Don't Know -5\%

2.Did the recommendation satisfied your feelings (mood)?

a.Positive Answer- $65 \%$ b.Negative Answer $-30 \%$ c.Don't Know $-5 \%$

3.Did the recommendation system was adapting to the different situations smoothly? a.Positive Answer- 76\% b.Negative Answer-24\% c.Don't Know - 0\%

4.Did the recommendation system recommended wrong services? a.Positive Answer- $86 \%$ b.Negative Answer -10\% c.Don't Know $-4 \%$

5. Did the recommendation help you to make a satisfactory decision? a.Positive Answer- $85 \%$ b.Negative Answer-12\% c.Don't Know-3\%

6. What rating you will give to the recommendation system based on its overall performance ? a.Very Good $-15 \%$ b.Good $-50 \%$ c.Average $-20 \%$ d. Not satisfactory $-15 \%$

7. Did the recommendation test help to prevent verbal fights ?

a.Satisfactory $-60 \%$ b. Moderately $-40 \%$ d. $0 \%$

8.Did the recommendation system always favored the member with high social dominance? a.Yes always- $10 \%$ b.Not always $-40 \%$ c. Judiciously $-50 \%$ d.Don't know $0 \%$ 


\section{CONCLUSION AND FUTURE WORK}

The users usually waste time in searching the suitable program as per his mood and other context. So many times he will get compromised with whatever the program readily available to him even though more interesting programs are available in the box. The proposed recommendation system is an attempt to solve such problems by recommending the programs based on the user current context (time, mood, activity and social situation). It imposes the social understanding to context aware television. The proposed system is capable to adapt to the situation like presence of father, mother or parents in a group.

Depending on the social situation TV recommends the service .The system has been tested with small families wherein father is more dominant. The experimental analysis has been proved that even though the system initially prefers only the socially dominant user but as the time goes on (with the help of sufficient history of the family watching pattern) the differences in the satisfaction level of the user decreases slowly. The usage of fuzzy based Bayesian approach has increased the performance in terms of time and memory even though the average recommendation precision of the system does not cross $90 \%$. This is mainly due to the change in the users TV watching pattern regularly and also due to the inclusion of new TV programs and channels due course. As per the survey report the system has satisfied the children's more in individual as compared to their parents because of their few choice (only cartoons).As a future work we are working towards to generalize the idea to all categories of families and groups considering the different factors like time,activity,mood,location size ,culture , ethics ,language ,personal ,physiological and social.

\section{ACKNOWLEDGMENTS}

The authors are thankful to Bhanuprakash.H.P, final year $\mathrm{BE}(\mathrm{CSE})$ student (2010-2011) for his valuable contribution towards the implementation of this project

\section{REFERENCES}

[1] Min-Cheol Hwang, Le Thanh Ha, Seung-Kyun Kim, and Sung-Jea Ko, Senior member, IEEE . Department of Electronic Engineering, Korea University, Seoul, Korea, 14244-0763-X/07/\$20.00 C2007 IEEE "Real-Time Person Identification System for Intelligent Digital TV".

[2] Z. Yu and X. Zhou, "TV3P An Adaptive assistant for Personalized TV," IEEE Trans. Consum. Electron., Vol. 50, No. 1, pp. 393-399,2004

[3] H. Yoon and W. Woo, "Design and Implementation of a Universal Appliance Controller Based on Selective Interaction Modes,', IEEE Trans. Consum. Electron., Vol. 54, No. 4, pp. 1722-1729, 2008.

[4] Dewan:Sunil “WIRELESS TRANSLATION DEVICE” 200090215394 A1 August 9th 2009. United states patent application kind code

[5] Adrian C. North, David J. Hargreaves, and Jon J. Hargreaves, "Uses of music in everyday life," Music Perception, vol. 22, no. 1, pp. 41-77, 2004.

[6] Choonsung Shin and Woontack Woo, Member, IEEE "Socially Aware TV Program Recommender for Multiple
Viewers" Downloaded on August 9, 2009 at 01:42 from IEEEXplore..

[7] Doug Williams, Marian F Ursu,, Ian Kegel , “An Emergent Role for TV in Social Communication".

[8] Dr Daniel Chandler, Matthew Ruckwood, Mediated Communication "Will Interactive Television Change the Relationship Between the Viewer and the Television Set? “ , MC10120 10/01/2005.

[9] Doug Riecken , "Perosonalized views of personalization ," Comm.ACM ,vol 43,n08,2000.

[10] Muhammad Ashad Kabir ,Jun Han and Alan Colman , "Modeling and Coordianting Social Interactions in Pervasive Environments “,

[11] N.Datia ,J Moura-Pires .M.Cardoso ,H.Pita , “Temporal Patterns o fTV Watching For Portugues Viewers ".

[12] Joe Jeffrey ,'High Fidelity Mathematical Models of Social Systems “, Northen Illinois University.

[13] Ray van Brandenburg , Master Thesis Faculty of Electrical Engineering, Mathematics and Computer Science, Design and Analysis of Communication Systems (DACS),University of Twente" Towards multi-user personalized TV services, introducing combined RFID Digest authentication" ,Dec2007,Master thesis

[14] Paul Resnick ,Neophytos Iacovou ,Mitesh Suchak Peter Bergstorm ,and John Riedli ,"Grouplens : An open architecture for collaborative filtering of netnews , " in Proc.ACM Conf.Computer Supported Cooperative Work ,Chape Hill ,NC,USA ,1994,pp.175 -186.

[15] Annie Chen ,"Context aware collaborative filtering system : Predicting the user's preference in the ubiquitous computing environment ," in Location and Context Awareness,pp.244-253 .Springer ,2005

[16] Manos Papagelis, Dimitris Plexousakis," Qualitative analysis of user-based and item-based prediction algorithms for recommendation agent ",Engineering Applications of $\begin{array}{llll}\text { Artificial Intelligence } & 18 & \text { (2005) 781-789 }\end{array}$ "www.elsevier.com/locate/engappai

[17] Manos Papagelis1, 2, Ioannis Rousidis2, Dimitris Plexousakis1, 2, Elias Theoharopoulos3 , "Incremental Collaborative Filtering for Highly- Scalable Recommendation Algorithms", Work conducted at ICSFORTH

[18] SongJie Gong , “A Collaborative Filtering Recommendation Algorithm Based on User Clustering and Item Clustering “,JOURNAL OF SOFTWARE, VOL. 5, NO. 7, JULY 2010, (C) 2010 ACADEMY PUBLISHER,doi:10.4304/jsw.5.7.745-752

[19] Kyusik Park, Jongmoo Choi, and Donghee Lee , "A Single-Scaled Hybrid Filtering Method for IPTV Program Recommendation “ , INTERNATIONAL JOURNAL OF CIRCUITS, SYSTEMS AND SIGNAL PROCESSING, Issue 4, Volume 4, 2010

[20] J.J. Sandvig and Bamshad Mobasher and Robin Burke , "A Survey of Collaborative Recommendation and the Robustness of Model-Based Algorithms “, Bulletin of the IEEE Computer Society Technical Committee on Data Engineering, Copyright 2008 IEEE. 
[21] Michael Hahsler , "Developing and Testing Top-N Recommendation Algorithms for 0-1 Data using recommender lab “, February 27, 2011 ,

[22] Amancio Bouza, Gerald Reif, Abraham Bernstein , "Probabilistic Partial User Model Similarity for Collaborative Filtering”.

[23] SongJie Gong , “ A Collaborative Filtering Recommendation Algorithm Based on User Clustering and Item Clustering",Journal Of Software ,Vol .5.No 7,July2010.

[24] Manos Papgelis ,Dimitris Plexousakis , "Qualitative analysis of user based and item based prediction algorithms for recommendation agents", Engineering Applications of Artificial Intelligence 18(2005) 781-789 ,www.elsevier.com/locate/engappai

[25] Artur Lugmayr, Tampere University of Technology, Finland Alexandra Pohl, Berlin-Brandenburg (rbb) Innovationsprojekte, Germany Max Mühlhäuser, Technische Universitat Darmstädt, Germany Jan Kallenbach, Helsinki University of Technology, Finland Konstantinos Chorianopoulos, Bauhaus University of Weimar, Germany "Ambient Media and home Entertainment" Copyright (C) 2007, IGI Global

[26] Prof. Dr. Artur Lugmayr , Entertainment and Media Production Management Lab. (EMMi Lab.) Tampere University of Technology (TUT), Tampere Finland,keynote address, Connecting the Real World with the Ubiquitous Overlay in ambient Media ", Keynote address , SAME 2009 in conjunction with AmI 2009, Salzburg, Austria.

[27] Artur Lugmayr \& Thomas Risse \& Bjoern Stockleben \& Kari Laurila \& Juha Kaario , "Semantic ambient mediaan introduction ", Multimed Tools Appl (2009) 44:337359 DOI 10.1007/s11042-009-0282-z , Published online: 6 May 2009 \# Springer Science + Business Media, LLC 2009

[28] L.Ardissono, C.Gena ,P.Torasso , “Personalized Recommendation of TV Progams".

[29] Soha Maad, Fraunhofer, Institute For Media Communication (IMK),Germany .Soha.Maad@imk.fhg.de "MARILYN: A Novel Platform For Intelligent Interactive TV (IITV)".

[30] Zhiwen Yu ,Xingshe Zhou , Yanbin HAo ,Jianhua Gu , “ TV program recommendation for multiple viewers based on user profile merging “, 16 April 2006 /Published online :10 June 2006 @ Springer Science Science + Business Media B.V.2006,User Model User Adap Iter (2006) 16 :63 -82 .

[31] Choonsung Shin and Woontack Woo," Conflict Resolution based on User Preference and Service Profile for Context aware Media Services “.

[32] Choonsung Shin and Woontack Woo, " Conflict Management for Media Services by exploiting Service Profile and User Preference “,UbiPCMM 2005,pp 48 -57

[33] Choonsung Shin and Woontack Woo , History based Conflict Management for Multiuser and Multi services ",

[34] Choonsung Shin and Woontack Woo, "User - Centric Conflict Management for Media Services Using Personal
Companions “, ETRI Journal, Volume 29, Number 3, June 2007.

[35] B.I.J. Siljee, I.E. Bosloper, J.A.G. Nijhuis , University of Groningen, Department of Computing Science PO Box 800, 9700 AV Groningen, The Netherlands \{b.i.j.siljee, i.e.bosloper, j.a.g.nijhuis\}@cs.rug.nl, ” A Classification Framework for Storage and Retrieval of Context " .

[36] Huadong Wu , "Sensor Data Fusion for Context-Aware Computing Using Dempster-Shafer Theory “,The Robotics Institute Carnegie Mellon University Pittsburgh, Pennsylvania 15213 December 2003.

[37] Pravin Pawar, Andrew Tokmakoff ,’Ontology based Context Aware Service Discovery for Pervasive environments".

[38] Jarno Seppanen, Jyri Huopaniemi , "Interactive and Context Aware Mobile Music Experiences “, Proc of the 11th Int Conference on Digital Audio Effects (DAFx08),Espoo, Finland ,September 1-4 ,2008.

[39] Anind k.Dey and Gregory D Abowd ,"Towards a better understanding of context and context awareness," Tech.Report GIT-GVU-99-22, Georgia Institute of Tech ., Atlanta, GA ,USA,1999.

[40] T. Strang and C. Linnhoff-Popien "A Context Modeling survey", Inthe first International Workshop on Advanced context modeling, Reasoning and management, UbiComp 2004.

[41] Moeiz Miraoui, Chakib Tadj and Chokri ben Amar, “ Context Modeling and Context Aware Service Adaptation for pervasive computing systems", International Journal of Computer and Information Science and Engineering 2008,pp $143-152$.

[42] B.N., Schilit .A context aware system architecture for mobile distributed computing $\mathrm{PhD}$ thesis, 1995.

[43] D.Dubois and H.Parade ,"An introduction to fuzzy systems,",Clin Chim Acta ,vol 270,pp-3-29,1998.

[44] Han-Saem Park ,Ji-Oh Yoo and Sung- Bae Cho “ A Context Aware Music Recommendation System Using Fuzzy Bayesian Networks with Utility Theory ",L.Wang et al.(EDs) :FSKD 2006 ,LNAI 4223 , pp. 970 -979,2006,@ Springer- Verlag Berlin Heidelberg 2006.

[45] H.pan and L.Liu ,'Fuzzy Bayesian networks : A general formalism for representation, inference and learning with hybrid Bayesian networks ," Int J Pattern Recognition ,vol.14,pp 941-962,2000.

[46] Thyagaraju.GS,U.P.Kulkarni,"Modelling Of User Preferences in Single and Multiuser Context Aware Environments For Interactive Context Aware TV", International Journal of Information Technology and Information Engineering ,IJITIE ,ISSN 0974 -4959, Volume 01 ,Issue No 01 , January 2011 - March 2011.

[47] Thyagaraju.GS,U.P.Kulkarni , , Interactive Democratic Group Preference Algorithm for Interactive Context Aware TV", 2010 IEEE International Conference on Computational Intelligence and Computing Research ,

[48] Thyagaraju.GS, U.P.Kulkarni” Modeling User Context for Interactive Context Aware TV", 2010 IEEE International 
Conference on Computational Intelligence and Computing Research .

[49] Thyagaraju.GS,U.P.Kulkarni "Conflict Resolving Algorithms to Resolve Conflict in Multi-user ContextAware Environments", 2009 IEEE International Advance Computing Conference (IACC 2009)Patiala, India, 6-7 March 2009 , 978-1T-4244-1888-6/08/

[50] Thyagaraju.GS , UP Kulkarni ,"Conflict Resolution in multiuser context aware environment", April /June 2008, http://doi.ieeecomputersociety.org/10.1109/MPRV.2008.25 , IEEE Pervasive Journal volume $=\{7\}$, number $=\{2\}$, issn $=\{1536-1268\}$, year $=\{2008\},($ Impact Factor 2.293)

\section{AUTHORS PROFILE}

Thyagaraju.GS received the M.Tech Degree in Computer Science And Technology From University Of Mysore ,India in 2002.He has got ten years of experience in academics ,fours years of Research Experience. He is a member of IETE.He has guided many students at UG and PG level.He is pursuing Ph.D in Computer Science Engineering. His Research Interests are Context Aware Computing in Ubiquitous and Intelligent Systems $\mathrm{He}$ is now working as a Senior Lecturer in Dept Of CSE, SDM College Of Engineering, Karnataka, Dharwad.

Dr. Umakant Kulkarni obtained his BE Degree from Karnataka University, Dharwad in the year 1989, ME Degree from PSG College of Technology, Coimbatore in the year 1991 and $\mathrm{PhD}$ from Shivaji University, Kolhapur in the year 2007. He has published many papers at International Journal and IEEE conferences in the areas of Pervasive and Ubiquitous Computing, Distributed Data Mining, Agents Technology and Autonomic Computing. He is Member of IETE and ISTE. He served as Head of Department and Chief Nodal Officer- TEQIP a World Bank funded project. He has guided many students at PG level and five research scholars are pursuing their PhDs. Currently he is serving as professor in the Department of Computer Science \& Engineering, SDM College of Engineering \& Technology, Dharwad, Karnataka State , India. 